\title{
APORTE DE LA QUÍMICA ANALÍTICA \\ A LA FORMACIÓN CIENTÍFICA DE LOS PROFESORES DE QUÍMICA
}

\author{
Carlos Arturo Franco Ruiz, Luis Enrique Salcedo Torres Ph. D.
}

Universidad Pedagógica Nacional.

\begin{abstract}
In this work it presented the results obtained when experiencing to pedagogic strategy based on the resolution of problems in Analytic Chemistry with the propose of contributing to the scientific formation of future professors of chemistry Evolution is presented in the conceptions of Science, investigation and scientific work, as well as in the key aspects of the resolution of analytic problems: hypothesis emission, design of experiments, identification of the analytic problems, handling information. They don't advance significantly in the delimitation of the field of validity of results.
\end{abstract}

\section{RESUMEN}

En este trabajo se presentan los resultados obtenidos al experimentar una estrategia pedagógica basada en la resolución de problemas en Química Analítica, con el fin de contribuir a la formación científica de futuros profesores de Química.

Se presenta evolución en las concepciones de Ciencia, Investigación y trabajo científico, así como en los aspectos claves de la resolución de problemas analíticos: emisión de hipótesis, diseño de experimentos, identificación de problemas analíticos y manejo de la información. No se avanza significativamente en la delimitación del campo de validez de resultados.

\section{INTRODUCCIÓN}

Estudios realizados de cómo aprenden los alumnos la ciencia, han confluido en afirmar que el estudiante construye significados entre sus conocimientos previos y el nuevo conocimiento (Ausubel, 1978). El estudiante es visto como alguien activamente implicado en la construcción de significados, aportando sus concepciones previas a la interpretación de nuevas situaciones. De esta manera, los profesores de ciencias deberían tener en cuenta estos resultados de la investigación didáctica, para procurar una correcta adquisición de los conocimientos científicos en sus alumnos, acompañada de su consecuente cambio metodológico (Gil 1985). Otras investigaciones han mostrado que así como los alumnos poseen preconcepciones, los profesores de ciencias, tienen sus 
propias concepciones (Hodson, 1986,1988), sobre la ciencia, la enseñanza y sobre el aprendizaje (Hewson, 1987), que influyen de una u otra manera en su actuación en el aula y que pueden entrar en conflicto con los resultados obtenidos en la investigación educativa.

Frente a este panorama, la formación inicial del profesorado de ciencias, en particular el de Química, constituye un aspecto clave para tener en cuenta, si se quieren posibilitar los cambios necesarios para dar respuesta a un mejoramiento cualitativo de la enseñanza y del aprendizaje de las ciencias.

En particular, en lo que atañe a la formación del profesorado de química, se hace necesario experimentar propuestas pedagógicas que integren coherentemente los aportes recientes de la filosofía de la ciencia y de la Sicología cognitiva con el ánimo de producir los cambios conceptuales, metodológicos, actitudinales y didácticos (Gil, 1986) necesarios para que su labor docente futura pueda favorecer el enriquecimiento espiritual, cultural y científico en sus alumnos.

Con este trabajo se espera contribuir a la formación científica del futuro profesor de Química, mediante la vivencia de procesos de producción del conocimiento científico a través de la resolución de situaciones problemáticas en el campo de la Química Analítica.

La formación científica se refiere a promover un verdadero espíritu de investigación y de pensamiento ordenado, proporcionar la máxima participación al estudiante con la subsiguiente motivación, favorecer la participación activa de los (as) estudiantes tanto en el planteamiento como en la ejecución del trabajo experimental, alentar a los (as) estudiantes a formular y aceptar críticas, proporcionar un ambiente donde el máximo nivel de trabajo no conozca otra limitación que la capacidad de los propios estudiantes, su entusiasmo y el tiempo disponible, desarrollar bases sólidas para poder afrontar las situaciones técnicas de la vida real, suministrar espacios para la discusión general y enseñar el uso correcto de la bibliografía y demás fuentes de consulta.

\section{LA QUÍMICA ANALÍTICA COMO CIENCIA}

¿Cómo se produce el conocimiento en Química Analítica? Normalmente, la producción del conocimiento en Química Analítica se realiza al buscar soluciones a problemas relacionados con la composición de los diversos materiales tanto naturales como sintéticos; es decir, al intentar identificar y cuantificar las sustancias químicas presentes en dichos materiales. Lo anterior con lleva a buscar algunas propiedades relacionadas con la naturaleza de las sustancias ya crear un instrumento adecuado para su medición; surgen así los métodos analíticos, que representan la confluencia entre el desarrollo científico y su aplicación tecnológica. Una vez creado el método, se estudian sus diversas aplicaciones a situaciones concretas y de esta manera surgen las técnicas analíticas que luego de ser estandarizadas pueden utilizarse indefinidamente, o bien extrapolarse a otro tipo de mediciones (Salcedo, 1994).

Se trata entonces de suministrar a nuestros (as) alumnos (as) oportunidades para examinar e investigar hechos y fenómenos por si mismos, situaciones que les permitan reconocer y plantear un problema analítico, el diseñar y realizar experimentos controlados, recoger y organizar información, identificar regularidades, formular hipótesis y teorías, 
evaluar evidencias y extraer conclusiones (Bastida, 1990) para de esta manera contribuir a la formación científica.

\section{MODELO DE ENSEÑANZA APRENDIZAJE POR INVESTIGACION}

Bajo este contexto se aplica el modelo de enseñanza y aprendizaje como investigación (Gil, 1991), cuya idea fundamental es plantearle a los alumnos una serie de actividades, que los motiven y los impulsen a resolver situaciones problemáticas, posibilitando de esta manera la vivencia de la investigación como una forma de aprendizaje; dichas actividades deben ser cuidadosamente preparadas y orientadas por el profesor, se resumen así:

Plantear situaciones problemáticas que -teniendo en cuenta las ideas, visión del mundo, destrezas y actitudes de los alumnos y alumnas-generen interés y proporcionen una concepción preliminar de las tareas.

- Proponer al estudiante el estudio cualitativo de dichas situaciones junto con la toma de decisiones para abordar problemas precisos (ocasión para que comiencen a explicitar funcionalmente sus ideas).

- Orientar el tratamiento científico de los problemas planteados, lo que conlleva entre otros:

— La invención de conceptos y emisión de hipótesis (se hacen predicciones a partir de las ideas previas).

- La elaboración de estrategias de resolución (incluyendo en su caso, diseños experimentales) para la contrastación de las hipótesis a la luz del cuerpo de conocimientos de que se dispone.

— La resolución y el análisis de los resultados, cotejándolos con los obtenidos por otros grupos de alumnos y por la comunidad científica.

- Plantear el manejo de los nuevos conocimientos a variedad de situaciones, para hacer posible la profundización y afianzamiento de los mismos, poniendo énfasis especial en las relaciones ciencia / técnica / sociedad que enmarcan el desarrollo científico, mostrando así el carácter de cuerpo coherente que tiene toda ciencia.

\section{PROBLEMA A RESOLVER}

Las prácticas de Laboratorio de Química Analítica, se reducen a la repetición mecánica de técnicas analíticas ya establecidas, y esta situación se relaciona con una deficiente comprensión de la naturaleza de la Química Analítica como ciencia experimental y en el predominio de una enseñanza-aprendizaje por transmisión-recepción de conocimientos ya elaborados.

¿Contribuye lo anterior a la formación científica de los profesores de Química?

\section{OBJETIVO}

Contribuir a la formación científica de los futuros profesores de Química a través de la experimentación de una propuesta pedagógica, basada en el modelo enseñanza aprendizaje por investigación. 


\section{HIPÓTESIS}

Un programa guía de actividades basado en la resolución de problemas analíticos favorece la formación científica de los profesores de Química.

\section{ASPECTOS METODOLÓGICOS}

La población esta conformada por estudiantes de los cursos de Química Analítica. Se experimentó con 12 alumnos (as) de V semestre de Licenciatura en Química en el curso de Analítica 1 durante el primer semestre de 1998, un programa guía de actividades durante 9 semanas de 8 horas c/u. (anexo 3).

Con el fin de caracterizar las ideas iniciales de los estudiantes "SOBRE CIENCIA, INVESTIGACIÓN Y TRABAJO CIENTIFICO" $Y$ "HABILIDADES PARA EL APRENDIZAJE CIENTIFICO" se aplicaron los documentos. No. 1 y No. 2 (anexos 1 y 2 ).

El documento No. 2 fue adaptado de un estudio realizado en la Universidad de Sapienza (Bandiera y otros, 1995).

Con el fin de identificar aspectos claves de la formación científica, se utilizó un artículo sobre la determinación de cafeína por espectroscopia ultravioleta en un analgésico. La labor del estudiante consistió en reconocer en el texto los aspectos característicos del trabajo científico. Para observar el cambio y la evolución en la identificación de tales aspectos se aplicó como pos test un artículo sobre la determinación de ácido acetil salicílico (AAS) por espectroscopia ultravioleta.

\section{RESULTADOS Y ANALISIS SOBRE LACONCEPCION DE CIENCIA, INVESTIGACIÓN Y TRABAJO CIENTÍFICO}
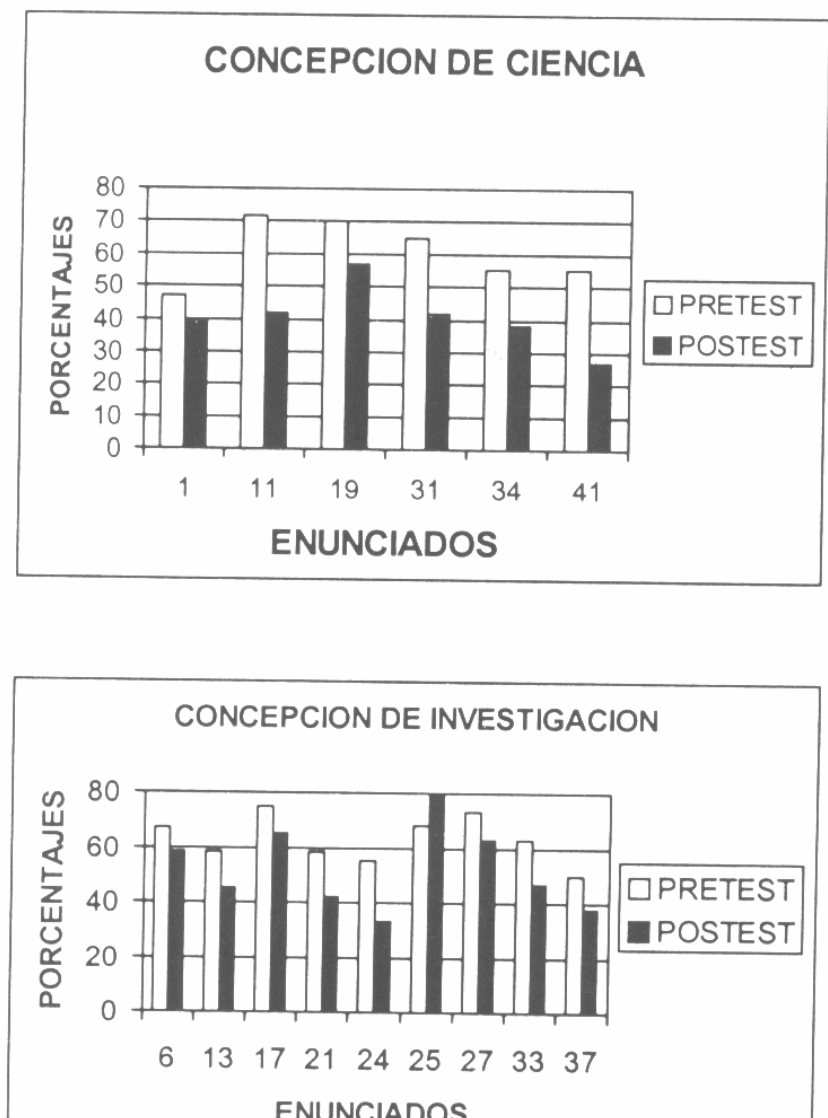
Los estudiantes cambiaron su concepción de ciencia, que estaba enmarcada como empiro positivista y asumen ahora una posición diferente de la ciencia. Los estudiantes ya no consideran la prevalencia de verdades absolutas, consideran que el conocimiento se construye y no se transmite; están de acuerdo que el papel del profesor va más allá de la transmisión del conocimiento. Siguen aceptando la idea que la ciencia evoluciona con la acumulación de teorías y que en su metodología se parte de la observación.

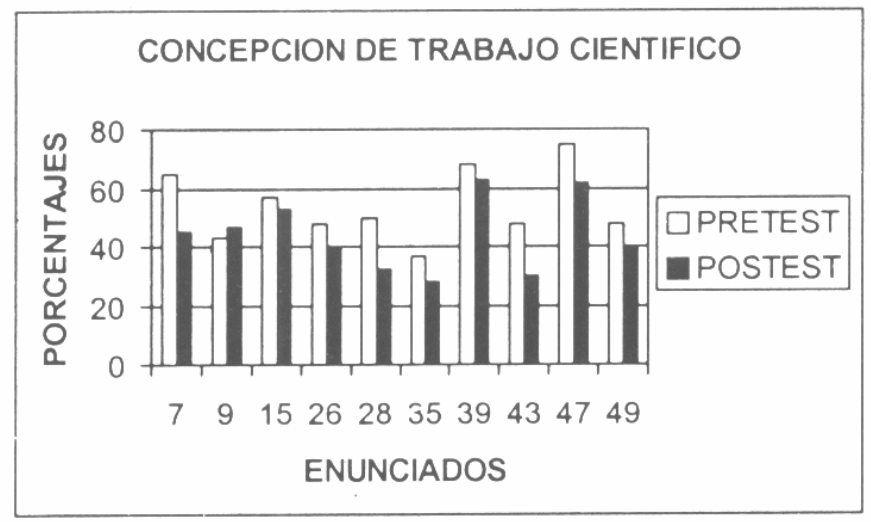

Respecto a la concepción de investigación, el cambio más significativo se corresponde con la aceptación de la idea que "los colectivos participan en el progreso de la ciencia", Realiza trabajo deductivo prevaleciendo y reconociendo la importancia del trabajo en grupos y la existencia de comunidades de especialistas. Descartan la posibilidad de que el investigador descubre la verdad.

Sobre la concepción de trabajo científico. El cambio más significativo se produce al aceptar y reconocer que bajo la expectativa del aprendizaje por investigación el trabajo científico está muy ligado al planteamiento y resolución de problemas.

\section{SOBRE LAS HABILIDADES PARA EL APRENDIZAJE CIENTÍFICO}

Los estudios comparativos en la pregunta de transposición de fórmula pasa de 2 a 8 estudiantes que responden correctamente y de 7 estudiantes que respondieron erróneamente en el pre test solamente 4 no lograron asimilarlo en el pos test. Con respecto a la presentación de gráficos, de 4 estudiantes que no respondieron y 5 que grafican sin restricciones, los 12 estudiantes grafican en el pos test, 5 de los cuales en forma correcta.

DEL LENGUAJE VERBAL AL LENGUAJE ALGEBRAICO

\begin{tabular}{|l|c|c|}
\hline & Pre test & Pos test \\
\hline No proporciona correctamente & 7 & 4 \\
\hline No reconoce proporcionalmente & 8 & 0 \\
\hline No involucra constante & 10 & 0 \\
\hline Reconoce criterio de proporcionalidad & 3 & 8 \\
\hline
\end{tabular}




\begin{tabular}{|l|l|l|}
\hline Involucra constante & 2 & 6 \\
\hline Realiza trabajo deductivo o analítico & 2 & 0 \\
\hline
\end{tabular}

DEL LENGUAJE VERBAL AL LENGUAJE GRAFICO

\begin{tabular}{|l|c|c|}
\hline & Pre test & Pos test \\
\hline No reconoce variables & 11 & 7 \\
\hline No gradúa ejes & 10 & 12 \\
\hline Grafica sin acotamientos & 8 & 12 \\
\hline Reconoce variables & 1 & 5 \\
\hline Gradúa ejes coordenados & 2 & 0 \\
\hline Grafica con restrincciones & 0 & 1 \\
\hline No grafica & 4 & 0 \\
\hline
\end{tabular}

Para la interpretación de la fórmula de Coulomb en donde 9 estudiantes respondieron teniendo diferente criterio, se pasó a 11 estudiantes que analizan la fórmula de una manera descriptiva.

De la misma manera, cuando se les solicita interpretar si es adecuada o no la fórmula planteada en la primera prueba 10 estudiantes realizan interpretación numérica mientras que en el pos test uno responde con criterio emotivo, tres analíticamente y 8 utilizan razones o análisis descriptivos.

La interpretación del gráfico complejo, mejoró notablemente, pues mejoraron las relaciones entre puntos y datos, hacen extrapolaciones, comparan los datos del gráfico y mejor aún, realizan con buena aceptación las inferencias pertinentes. En cuanto a la lectura de datos experimentales, la mayoría hace interpretaciones globales o elaboraciones lógicas. Se nota evolución al generar conclusiones, ya que se pasa de tres a ocho estudiantes que concluyen algún (os) aspecto (s) para datos experimentales.

\section{DEL LENGUAJE ALGEBRÁICO AL LENGUAJE VERBAL}

\begin{tabular}{|l|c|c|}
\hline & Pre test & Pos test \\
\hline Descriptivo & 2 & 0 \\
\hline Analítico & 6 & 11 \\
\hline Sumariamente & 1 & 0 \\
\hline Incompleto & 2 & 1 \\
\hline No respondió & 1 & 0 \\
\hline$x$ & & \\
\hline$x$ & & \\
\hline
\end{tabular}

\begin{tabular}{|l|c|c|}
\hline \multicolumn{3}{|c|}{ JUICIOS DE VALOR } \\
\hline & Pre test & Pos test \\
\hline Numérico & 10 & 0 \\
\hline Socioeconómico & 0 & 0 \\
\hline Emotivo (justicia) & 1 & 1 \\
\hline
\end{tabular}




\begin{tabular}{|l|l|l|}
\hline Analítico $(\mathrm{R}=0)$ & 0 & 3 \\
\hline Propone cambio (analiza) & 4 & 3 \\
\hline No responde & 1 & 1 \\
\hline Descriptivo & 0 & 1 \\
\hline
\end{tabular}

Los estudiantes mejoran su capacidad de interpretación en cuanto al manejo de fórmulas, construcción, lectura e interpretación de gráficos y análisis de datos experimentales.

De los resultados se deduce que en el pre test el $100 \%$ de los estudiantes no reconoce adecuadamente $\mathrm{o}$ no resalta los aspectos involucrados en un trabajo científico. Por el contrario, en el pos test, 8 estudiantes en promedio reconocen o resaltan del texto los aspectos inherentes al trabajo científico. Solamente se nota un cambio poco favorable en cuanto a reconocer que los resultados se deben confrontar con los reportados en la literatura.

Se presentan dificultades en la formulación de hipótesis y en poder extrapolar los resultados y procedimientos a otros tipos de situaciones.

En lo referente a los tres informes de actividades experimentales que ellos elaboraron individualmente o por parejas durante el desarrollo de la estrategia, se puede concluir que:

En el primer informe hubo dificultades en caracterizar cada uno de los aspectos claves del trabajo científico; sin embargo, para el segundo informe, los estudiantes plantearon problemas claros, referenciaron aspectos teóricos, involucraron hipótesis y variables y a la vez hicieron interpretación de los datos. No incluyeron comentarios sobre la confrontación de los resultados, no tuvieron en cuenta la validez de los resultados, como tampoco incluyeron nuevos problemas ni le dieron aplicación a situaciones concretas.

Para el tercer informe hay avance en la mayoría de los aspectos, pero tienen dificultades tanto en la aplicabilidad de los resultados como en la validez y confiabilidad de los mismos.

SOBRE EL PROGRAMA GUÍA DE ACTIVIDADES. Aunque los estudiantes manifiestan con agrado la vivencia de este otro enfoque y muestran interés para que se siga aplicando esta metodología en las nuevas disciplinas por cursar, se nota todavía cierta dependencia del maestro y poca autonomía para realizar y desarrollar sus compromisos propios de la asignatura.

La estrategia fue aceptada y tuvo buen margen de efectividad en su ejecución. Con la aplicación del modelo de enseñanza y aprendizaje por investigación, se logró poner en conflicto cognitivo a los estudiantes y más importante aún, se logró que ellos vivenciaran un periodo de crisis por el nuevo modelo presentado.

Algunas valoraciones hechas por los estudiantes a la estrategia se presentan a continuación:

- Se pudo entrar en confrontación con el modelo tradicional de transmisión.

- Permite cambiar la actitud frente al papel del experimento. 
- Permite comprometerse consigo mismos y delimitar sus propios criterios de responsabilidad.

- Permite trabajar ya sea en ausencia o en presencia del profesor.

- Permite vivenciar situaciones propias del trabajo científico.

- Permite explicitar las ideas propias y abrir espacios para la creatividad y la producción personal.

Al proponerle a los estudiantes la realización de un ensayo individual que involucrara algunos conceptos tratados durante la estrategia, se evidencia en ellos un efecto positivo de la estrategia como quiera que se nota reflexión crítica fundamentada; se logró que el estudiante se hiciera consciente de su propia elaboración y a la vez pudieran realizar escritos o informes sobre determinado contenido.

La elaboración, realización, ejecución y análisis de diseños experimentales permitió vivenciar el trabajo que realizan los Químicos Analíticos.

A la luz de las hipótesis se notó cambio favorable en los estudiantes como resultado de la aplicación de la estrategia.

\section{CONCLUSIONES}

- Los estudiantes no están familiarizados con la interpretación de fórmulas y datos experimentales, se les dificulta la construcción adecuada de gráficos coordenados.

- Se contribuye significativamente a la formación científica de los estudiantes, haciendo que el planteamiento y resolución de problemas analíticos se constituya en la oportunidad de vivenciar las características del trabajo científico, tales como: la formulación de problemas, la emisión de hipótesis, el diseño de experimentos, el análisis crítico, la confrontación de resultados, etc.

- La aplicación de la estrategia mostró un cambio positivo hacia la concepción de ciencia y del trabajo científico y lo que debería ser una investigación en Química Analítica.

- El problema y las hipótesis fundamentadas se constituyen en aspectos relevantes de la estrategia en la medida que orientan la actividad experimental.

- La delimitación del campo de validez de la actividad experimental y los resultados obtenidos son aspectos del trabajo científico con el cual los alumnos no avanzan significativamente.

- Los estudiantes al reconocer el modelo propuesto manifiestan que es una alternativa de cambio y que dicha herramienta les permite reconocerse a sí mismos y a la vez les da oportunidad construir sus propios significados y acercarse de hecho a un trabajo de investigación y a la misma labor científica.

\section{RECOMENDACIONES}


- Se debe profundizar en las inferencias que se pueden hacer con los resultados experimentales, las aplicaciones a otras situaciones, el planteamiento de nuevos problemas y la consulta bibliográfica.

- Se recomienda que el programa guía de actividades no solamente se utilice como una estrategia metodológica, sino como recurso útil para evaluar y mejorar los procesos de enseñanza aprendizaje de las ciencias.

\section{BIBLIOGRAFÍA}

1. AUSUBEL P.D., 1978. Psicología Educativa: un punto de vista cognoscitivo (Trillas, México).

2. BANDIERA, M., Dupré, F., lanniello, M. G. y Vicentini, M., 1995. Una investigación sobre habilidades para el aprendizaje científico. Enseñanza de las ciencias. 13(1), pp. 46-54.

3. BASTIDA, M.F., Ramos, F.,Soto, J., 1990. Prácticas de laboratorio: ¿Una inversión poco rentable? Investigación en la escuela, 11, pp. 77-91.

4. GIL D., Carrascosa J., 1985. Science Learning as a conceptual a methodological Change. European Journal of Science Education, 7(3), pp. 231-236.

5. GIL, D., 1986. La metodología científica y la enseñanza de las ciencias: unas relaciones controvertidas. Enseñanza de las ciencias, 4(2), pp. 111-121.

6. GIL D., Carrascosa J., Furió C., Martínez-Torregrosa J., 1991. La enseñanza de las ciencias en la educación secundaria. CE. Universidad de Barcelona. (Editorial Horsiri), pp. 28.

7. GIL PÉREZ, D., 1991. Qué hemos de saber y saber hacer los profesores de ciencias. Enseñanza de las ciencias, 9(1), pp. 69-77.

8. GIL D., y Martínez Torregrosa J., 1987. Los programas guía de actividades: una concreción del modelo constructivista de aprendizaje de las ciencias. Investigación en la escuela, 3, pp. 3-12.

9. HEWSON P., and Hewson M., 1987. Science teachers Conception of teaching: implications for teacher education. International Journal of science Education. Vol. 9(4) pp. 425-440.

10.HODSON D., 1986. Philosophy of science and the science Curriculum. Journal of Philosophy of Education. Vol. 20 pp. 241-251

11.HODSON D., 1988. Toward a philosophy more valid Science Curriculum. science Education, vol. 72(4), pp. 19-40.

12. SALCEDO L., 1994. Contribuciones de la Química Analítica a la Formación Científica de los profesores de Química. Proyecto de investigación aprobado por la facultad de Ciencia y Tecnología para el ascenso a la categoría de profesor titular. U.RN. (Bogotá). 


\section{ANEXO No 1 \\ DOCUMENTO No 1 \\ CONCEPCIONES SOBRE CIENCIA, INVESTIGACIÓN Y TRABAJO CIENTÍFICO}

\section{INSTRUCCIONES}

A continuación encontrará una serie de enunciados numeradas de 1 a 50 , sobre las cuales se le solicita su posición, de conformidad con la escala:

1. TOTALMENTE EN DESACUERDO

2. EN DESACUERDO

3. SIN OPINIÓN

4. DEACUERDO

5. TOTALMENTE DE ACUERDO

Marque con una $X$ en las casillas correspondientes a cada afirmación, en la hoja de respuestas, la que corresponda a su parecer, según el orden en la escala. Por favor no escriba nada en este cuadernillo.

Los resultados serán utilizados con propósitos eminentemente investigativos, por lo que su nombre en la hoja de respuestas tendrá una función de control y no será mencionado bajo ninguna consideración.

\section{ENUNCIADOS}

1. La ciencia evolucione con el estudio y la acumulación de teorías.

2. El conocimiento se construye a través de la investigación.

3. Las hipótesis determinan las pautas para realizar los experimentos.

4. Los cambios de paradigmas son el resultado de teorías en conflicto.

5. Para el investigador, el experimento determina si les hipótesis se aceptan o se rechazan.

6. La investigación científica se caracteriza por el método científico.

7. Las Observaciones originan conocimiento científico.

8. Las hipótesis se pueden aceptar según el resultado de muchas investigaciones.

9. El conocimiento científico se logra a través de la acumulación de observaciones.

10. El observador deforma la realidad.

11. La ciencia ha evolucionado por acumulación sucesiva de verdades observadas en la naturaleza.

12. Las teorías científicas son el resultado de numerosas investigaciones.

13. Las teorías están en la naturaleza. 
14. El status del conocimiento científico genera situaciones cambiantes.

15. El conocimiento científico es verdadero.

16. Toda investigación científica comienza con el planteamiento de un problema.

17. Observación, experimentación, enunciado y comprobación de leyes y teorías. Representan el método científico.

18. Las investigaciones son programas que desarrollan las comunidades de especialistas.

19. La ciencia se caracteriza por su método inductivo que parte de la observación.

20. Cuando se habla de investigación científica, aparecen nuevos problemas.

21. Las hipótesis y las teorías científicas se construyen a partir de la observación.

22. Las hipótesis y teorías científicas se construyen a través de programas de investigación.

23. Los resultados de una investigación científica, originan nuevas situaciones para investigar.

24. Los colectivos obstruyen el progreso de las ciencias.

25. La investigación es un proceso que se realiza por etapas previamente establecidas.

26. Las observaciones que se realicen proporcionan conocimiento científico.

27. Toda investigación científica comienza por la observación sistemática del fenómeno que se estudie.

28. El crecimiento científico se logra por acumulación sucesiva de observaciones.

29. El conocimiento científico es el producto del trabajo en colectivos.

30. La ciencia se caracteriza por trabajar con programas de investigación.

31. Las teorías científicas son verdades sacadas de regularidades observadas en la naturaleza.

32. Las teorías se conciben bajo la estructura de una investigación científica.

33. En la observación se evita toda deformación de la realidad por parte del observador.

34. En la naturaleza hay verdades absolutas.

35. Las hipótesis son aceptadas por el investigador sin la necesidad de un trabajo experimental.

36. En el estudio de las ciencias el método científico se refiere a un programa de investigación.

37. En la investigación científica el investigador descubre la verdad.

38. Con los cambios en programas de investigación, la ciencia evolucione.

39. Los científicos actúan con un instrumento objetivo para formular sus teorías.

40. Cuando se plantean problemas, las hipótesis son el marco de referencia para que estas se contrasten.

41. El profesor transmite el conocimiento.

42. El investigador, para falsar una hipótesis, requiere de varios experimentos.

43. Los experimentos son desarrollos prácticos de verdades ya establecidas.

44. Se llega a un periodo de crisis en la ciencia cuando hay muchos enigmas por resolver.

45. El conocimiento científico es cambiante.

46. El experimento juega un papel fundamental en la investigación científica.

47. Las prácticas de laboratorio sirven para corroborar las teorías.

48. El trabajo de colectivos es una vía para poder contrastar las experiencias individuales.

49. Al desarrollar rigurosamente una guía de laboratorio, se puede acceder al conocimiento.

50. Los resultados de las investigaciones se deben contrastar con los reportados en la literatura. 


\section{ANEXO No. 2 \\ DOCUMENTO No. 2 \\ HABILIDADES PARA EL APRENDIZAJE CIENTÍFICO}

\section{NOMBRE:}

Lea cuidadosamente c/u de las preguntas y respóndalas individualmente. Si no sabe responder siga al punto siguiente.

Este cuestionario es eminentemente investigativo por lo que su nombre y sus respuestas serán de uso exclusivo del investigador.

1. La Sinfonanza (5) de un catorce (c) es directamente proporcional a la Alianza (P) del catorce, al Garone (G) e inversamente proporcional a la Trenza (T).

a. Expresarla afirmación mediante una fórmula algebraica.

b. Representar gráficamente la afirmación usando el/los gráficos convenientes.

2. La fuerza $F$ entre dos iones cargados respectivamente con cargas $Q_{1}$ y $Q_{2}$ colocados ala distancia $R$ se expresa mediante la fórmula $F=K Q_{1} Q_{2 / R}{ }^{2}$, donde $K$ es una constante.

Exprese en palabras el significado de la fórmula.

3. La Asociación Bogotana de amas de casa ha propuesto que el sueldo anual 5 de las amas de casa casadas sea calculado según la siguiente fórmula: $5=a n N / R$. Siendo a un factor preestablecido e igual para todas, $\mathrm{n}$ el número de hijos de la familia, $\mathrm{N}$ el número de habitaciones de la casa en que vive la familia y $R$ el valor monetario de los regalos recibidos del marido en un año.

a. Expresar en palabras el significado de la fórmula aunque no te parezca apropiada.

b. Si la fórmula no te parece apropiada específica las razones (puedes utilizar ejemplos).

4. Análisis de un gráfico.

En la figura se presentan los datos relativos al consumo anual de cigarrillos (-) y los casos de cáncer de pulmón (-) sobre 100.000 habitantes en Bogotá referidos a hombres (---) ya mujeres (---). Utilizando exclusivamente los datos presentados en el gráfico, clasifica las afirmaciones enumeradas en el cuadro adjunto señalando con una equis $(X)$ las que considere adecuadas.

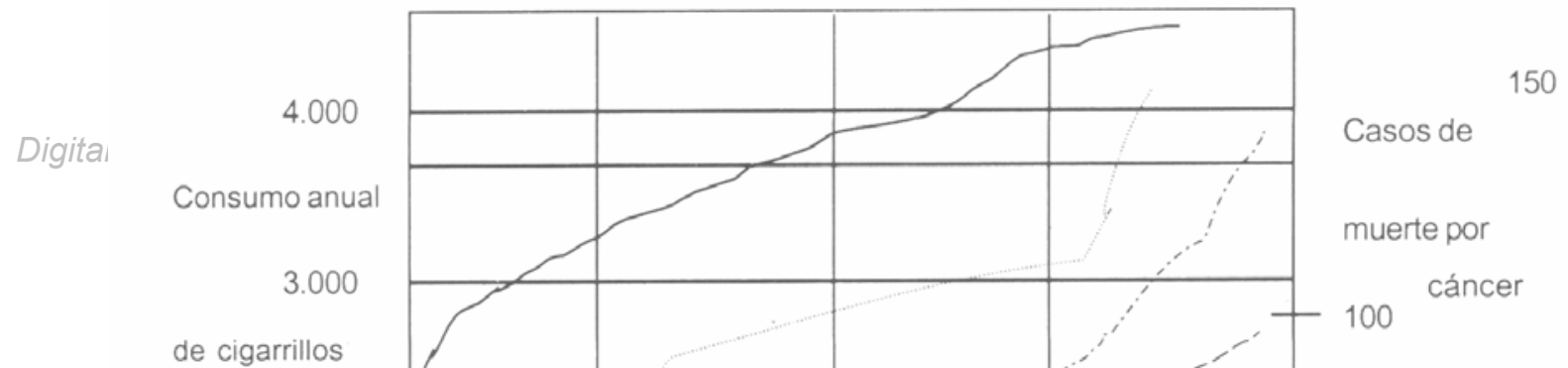


Para las afirmaciones referidas a los fenómenos observados en la gráfica anterior ténganse en cuenta las siguientes claves:

$\mathrm{V}$ Verdadera con base al texto

F Falsa con base al texto.

N No se deduce del texto.

A Aceptable.

ND Sin decisión

\begin{tabular}{|c|c|c|c|c|c|}
\hline Afirmaciones & $\mathbf{v}$ & $\mathbf{F}$ & $\mathbf{N}$ & A & ND \\
\hline 1. La recogida de los datos ha sido hecha cada veinte años. & & & & & \\
\hline 2. Antes de 1930 las mujeres bogotanas no morían de cáncer. & & & & & \\
\hline $\begin{array}{l}\text { 3. El cáncer pulmonar es provocado principalmente por el humo del } \\
\text { cigarrillo. }\end{array}$ & & & & & \\
\hline $\begin{array}{l}\text { 4. Se puede predecir que en } 1990 \text { no habrá más diferencia entre los } \\
\text { hombres y las mujeres bogotanas en el consumo de cigarrillos. }\end{array}$ & & & & & \\
\hline $\begin{array}{l}\text { 5. El consumo de cigarrillos conlleva el mismo riesgo de enfermarse } \\
\text { de cáncer pulmonar tanto para hombres como para mujeres }\end{array}$ & & & & & \\
\hline $\begin{array}{l}\text { 6. El consumo de cigarrillos por las mujeres bogotanas ha aumentado } \\
\text { aproximadamente cinco veces entre } 1940 \text { y } 1970 .\end{array}$ & & & & & \\
\hline $\begin{array}{l}\text { 7. Un hombre que fuma consume más cigarrillos que una mujer } \\
\text { fumadora. }\end{array}$ & & & & & \\
\hline $\begin{array}{l}\text { 8. En el caso de las mujeres, inciden otros factores en el número de } \\
\text { casos de cáncer pulmonar. }\end{array}$ & & & & & \\
\hline
\end{tabular}

\section{Lectura de datos experimentales}

Teniendo en cuenta la observación de que muchos organismos vivientes se encuentran sobre todo cerca o en el interior de sustancias orgánicas en descomposición (por ejemplo moscas de la fruta, gusanos y mufa en el queso, microorganismos en caldos e infusiones) algunos científicos han formulado la hipótesis de que estos organismos se originan espontáneamente a partir de esas sustancias.

Para verificar experimentalmente tal hipótesis se puede pensar en tratar de modos diferentes cuatro series de probetas conteniendo caldo de carne turbio (por la presencia de microorganismos vivos) y luego compararlas, haciendo un total de ocho grupos de observaciones. 
En la tabla siguiente se muestran los tipos de tratamiento (filas) y se indica el aspecto que presenta el caldo en las probetas, después de una hora y después de diez días de haber realizado el tratamiento, respectivamente (columnas). Las ocho casillas numeradas corresponden a las observaciones.

\begin{tabular}{|l|l|l|}
\hline \multicolumn{1}{|c|}{ TRATAMIENTOS } & \multicolumn{2}{c|}{$\begin{array}{c}\text { Aspecto del caldo después de } \\
1 \text { hora }\end{array}$} \\
\hline $\begin{array}{l}\text { Probetas cubiertas con una gasa. } \\
\text { Calentamiento durante 40 mm. a 60 C. }\end{array}$ & 1 turbio & 2 claro \\
\hline $\begin{array}{l}\text { Probetas cubiertas con una gasa. } \\
\text { Calentamiento durante 40 mm. A 100 C. }\end{array}$ & 3 claro & 4 turbio \\
\hline $\begin{array}{l}\text { Probetas selladas con cera. } \\
\text { Calentamiento durante 40 mm. A 60 C. }\end{array}$ & 5 turbio & 6 turbio \\
\hline $\begin{array}{l}\text { Probetas selladas con cera. } \\
\text { Calentamiento durante 40 mm. A 100 C. }\end{array}$ & 7 claro & 8 claro \\
\hline
\end{tabular}

Para interpretar estos datos se proponen las afirmaciones abajo enumeradas.

Indica para cada una de ellas si la consideras demostrada (D) o no demostradas (ND) de acuerdo con los datos (señala con una cruz la casilla correspondiente a tu evaluación).

\begin{tabular}{|c|c|c|}
\hline & $\mathbf{D}$ & ND \\
\hline 1. La generación espontánea es posible. & & \\
\hline 2. La generación espontánea no es posible. & & \\
\hline 3. El oxigeno no es necesario para la vida de los organismos en el caldo. & & \\
\hline 4. La gasa permite que entre aire en la probeta. & & \\
\hline 5. Los organismos presentes en el caldo no mueren a la temperatura de $60 \mathrm{C}$. & & \\
\hline 6. Para que se formen microorganismos en el caldo son suficientes 10 días. & & \\
\hline 7. Para obtener resultados significativos es necesario calentar durante $40 \mathrm{~mm}$. & & \\
\hline 8. Los organismos que enturbian el caldo no se originan a partir del caldo. & & \\
\hline 9. Los microorganismos se generan en el caldo como los gusanos en el queso. & & \\
\hline
\end{tabular}

Anexo No. 3

\section{PROGRAMA GUIA DE ACTIVIDADES}

1. Diligencie el DOCUMENTO No 1. "Concepciones sobre ciencia, Investigación y trabajo científico".

2. Diligencie el DOCUMENTO No. 2."Habilidades para el aprendizaje científico".

3. Analice el DOCUMENTO No. 3 "Ciencia, Investigación y Trabajo Científico". Comente el documento en pequeños grupos y confronte sus conclusiones con las propuestas de los demás grupos. Anexo No. 6. 
4. En forma individual y por escrito haga un listado de todo aquello que caracterice el trabajo científico.

Realice una puesta en común y sáquense las conclusiones del grupo.

5. Lea el DOCUMENTO No. 4 "El trabajo Experimental en Química Analítica y su relación con el Trabajo Científico": Determinación de cafeína por espectroscopia Ultra Violeta en una pastilla de mejoral.

Individualmente señale en el documento las características del trabajo científico de acuerdo a las conclusiones de la actividad No. 4. Anexo No. 7.

6. En pequeños grupos comparta las respuestas señaladas en el Documento No. 4 y realice una propuesta de grupo.

7. Lea y analice el DOCUMENTO No. 5. "La Química Analítica como Ciencia y su importancia".

Confronte la información del Documento con lo discutido en las actividades anteriores. Exponga sus comentarios e inquietudes para analizarlos a nivel general.

8. Diligencie el DOCUMENTO No. 6 "Valoración de aspectos del trabajo científico considerados en las actividades prácticas en Química Analítica".

9. Lea y analice en forma individual el DOCUMENTO No. 7 "Modelo Enseñanza Aprendizaje por investigación".

Plantee sus comentarios en forma general y entregue por escrito las conclusiones del modelo que resulten del análisis del grupo.

10. Una compañía de tratamiento de aguas, nos solícita determinar cuantitativamente el contenido de nitratos en muestras de agua para consumo. Según la solicitud, determine el problema a resolver.

11. ¿Qué variables y de qué tipo se podrían inferir (trabajar) en este problema?

12. Determinar algunas hipótesis de trabajo que se relacionen con el problema planteado.

13. En pequeños grupos y por escrito unifíquense las soluciones a las actividades 10 , $11 \mathrm{y} 12$.

14. Realizar una puesta en común y sacar las conclusiones del grupo sobre los cuestionamientos anteriores.

15. Según la solicitud, ¿qué método analítico debe emplearse?

Plantee en forma individual y por escrito su posible solución.

16. Hacer una puesta en común sobre los posibles métodos que se puedan utilizar, de acuerdo a las propuestas individuales.

17.Lea y analice el DOCUMENTO No. 8, "Métodos analíticos para la determinación de nitratos". Plantear conclusiones en forma general. Anexo No. 11.

18. Resuelva el DOCUMENTO No. 9, "Cuestionario sobre Espectrofotometría Ultravioleta". Consulte la bibliografía disponible y que considere necesaria.

19. Con base en el trabajo individual y la información analizada en las actividades anteriores, plantear en pequeños grupos una posible respuesta (hipótesis) al problema presentado en la actividad No. 10.

Establecer una HIPÓTESIS GENERAL con base en los aportes de cada grupo.

20.Por escrito y en grupo sugiera y describa un diseño experimental que permita contrastar la hipótesis formulada. Consulte la información bibliográfica que considere pertinente.

21.Mediante un esquema presente y explique a los demás grupos el procedimiento diseñado, indicando los materiales y reactivos mínimos necesarios.

22. Una vez elaborado el diseño con la participación de todos los grupos, proceder a su ejecución en cada grupo de trabajo.

23. Haga un barrido con uno de los patrones y determine el lamda máximo de absorción. Construya con los datos obtenidos las gráficas:

a. \% T Vs C de los patrones. 
b. A Vs C de los patrones.

c. La curva espectral (A Vs I).

Aplique el método de los mínimos cuadrados para la gráfica y elabore la curva de calibración corregida.

A partir de lo anterior determine la cantidad de nitratos en la muestra problema.

24. Determine la confiabilidad y la validez de los resultados.

25. Haciendo uso de tablas y gráficas, sustente y confronte los resultados (incluyendo su tratamiento matemático y estadístico) con los obtenidos por los demás grupos.

Con base en los resultados, se acepta o se rechaza la hipótesis formulada en la actividad No. 19? Justifique su respuesta.

26. Elabore y entregue un informe escrito que incluya los diversos aspectos del experimento realizado.

27. Se sabe que algunos medicamentos contienen sustancias activas que absorben en el ultravioleta. Uno de los más comunes es la aspirina, que en su esencia contiene cafeína. Un laboratorio farmacéutico requiere hacer el control de calidad para el lote de producción No A250 de pastillas de aspirina, cuyo peso neto por unidad es de $180 \mathrm{mg}$. Para su producción y expendio al público las unidades de aspirina deben contener 40 mg de cafeína por cada $180 \mathrm{mg}$ de preparado.

Según la solicitud, determine el problema a resolver.

28. ¿Qué variables y de qué tipo se podrían inferir (trabajar) en este problema?

29. Determinar algunas hipótesis de trabajo que se relacionen con el problema planteado.

30 . En pequeños grupos y por escrito unifíquense las soluciones a las actividades 27,28 , 29.

31. Realizar una puesta en común y sacar las conclusiones del grupo sobre los cuestionamientos anteriores.

32. Según la solicitud, ¿qué método analítico debe emplearse? Plantee en forma individual y por escrito su posible solución.

33. Hacer una puesta en común sobre los posibles métodos que se puedan utilizar, de acuerdo a las propuestas individuales.

34. Con base en el trabajo individual y la información analizada en las actividades anteriores, plantear en pequeños grupos una posible respuesta (hipótesis) al problema presentado en la actividad No. 27.

Establecer una HIPÓTESIS GENERAL con base en los aportes de cada grupo.

35.Por escrito yen grupo sugiera y describa un diseño experimental que permita contrastar la hipótesis formulada. Consulte la información bibliográfica que considere pertinente.

36. Mediante un esquema presente y explique a los demás grupos el procedimiento diseñado, indicando los materiales y reactivos necesarios.

37. Una vez elaborado el diseño con la participación de todos los grupos, proceder a su ejecución en cada grupo de trabajo.

38. Elabore patrones de cafeína y realice sus mediciones.

Construya con los datos obtenidos las gráficas:

a. \% T Vs $\mathrm{C}$ de los patrones

b. A Vs O de los patrones.

Aplique el método de los mínimos cuadrados para la gráfica y elabore la curva de calibración corregida.

A partir de lo anterior determine la cantidad de cafeína en una pastilla de mejoral.

39. Determine la confiabilidad y la validez de los resultados.

40. Haciendo uso de tablas y gráficas, sustente y confronte los resultados (incluyendo su tratamiento matemático y estadístico) con los obtenidos por los demás grupos. 
41. Elabore y entregue un informe escrito que incluya los diversos aspectos del experimento realizado.

42. Qué importancia tiene el experimento realizado y en qué otras situaciones se puede aplicar el procedimiento y resultados obtenidos.

43. Lea y comente el DOCUMENTO No.10. Técnicas para determinar $I_{\max }$ en el ultravioleta". Anexo No. 13.

44. Lea y comente el DOCUMENTO No. 11. "Absorción en el Ultravioleta". Desarrolle los ejercicios y confróntelos con los reportados en la literatura. Anexo No. 14.

45. Lea y comente el DOCUMENTO No. 12. Artículo publicado en una revista especializada.

46. Diligencie el DOCUMENTO No. 2, "Habilidades para el aprendizaje científico". Anexo No. 5.

47. Diligencie el DOCUMENTO No. 6. "valoración de aspectos del trabajo científico considerados en las actividades prácticas propuestas en química analítica". Anexo No. 9.

48. Diligencie el DOCUMENTO No. 13. "Modelos guía de laboratorios en Química Analítica". Anexo No. 16.

49. Diligencie el DOCUMENTO No. 1. "Concepciones sobre ciencia, investigación y trabajo científico". Anexo No. 4.

50. Diligencie el DOCUMENTO No. 14. "El trabajo experimental en química analítica y su relación con el trabajo científico»". Anexo No. 17.

51. Elabore un escrito (ponencia o ensayo) sobre el tema Espectrofotometría Ultravioleta, considerando términos como: Métodos ópticos de análisis, radiación, patrones, transiciones electrónicas, lambda máximo, regresión, frecuencia, $h$, ley de Beer, exactitud, precisión etc. Adicione los términos que considere necesarios incluyendo ejemplos. 